\title{
The predicted metallicity distribution of stars in dwarf spheroidal galaxies
}

\author{
Gustavo A. Lanfranchi ${ }^{1}$ and Francesca Matteucci ${ }^{2}$ \\ ${ }^{1}$ IAG-USP, R. do Matão 1226, Cidade Universitária, 05508-900 São Paulo, SP, Brazil \\ email: glanfran@usp.br \\ ${ }^{2}$ Dipartimento di Astronomia-Universitá di Trieste, Via G. B. Tiepolo 11, 34131 Trieste, Italy \\ email: matteucci@ts.astro.it
}

\begin{abstract}
We predict the metallicity distribution of stars for 6 Dwarf Spheroidal (dSph) galaxies of the Local Group by means of a chemical evolution model which is able to reproduce several observed abundance ratios and the present day total mass and gas content of these galaxies. The model adopts up to date nucleosynthesis and takes into account the role played by supernovae of different types (II, Ia). Each galaxy model is specified by the prescriptions of the star formation rate and by the galactic wind efficiency chosen to reproduce the main features of these galaxies.
\end{abstract}

\section{The Models}

We use the chemical evolution model for dSphs of Lanfranchi \& Matteucci (2003), in which the dSph galaxies are characterized by one or two long episodes of star formation (SF) and by the occurrence of very intense galactic winds. The number, epoch and duration of the episodes are given by the SF histories inferred by CMDs. The main characteristcs of the models are described in Lanfranchi \& Matteucci $(2003,2004)$.

\section{Results}

\subsection{The $[\alpha / F e]$ ratio}

In figure 1 (left side), the $[\alpha / \mathrm{Fe}]$ ratios as functions of $[\mathrm{Fe} / \mathrm{H}]$ are shown in comparison with the predictions of models for each of the $6 \mathrm{dSph}$ galaxies. The solid lines correspond to the best model for each galaxy while the dashed lines represent the lower and upper limits for the SF efficiencies. As one can see, the models reproduce fairly well all the abundance ratios in every galaxy, in the ranges of SF and wind efficiencies given by the models (for more details see Lanfranchi \& Matteucci 2004). The sudden decrease in the predicted abundance ratios, consistent with the observed data, is a consequence of the occurrence of galactic winds and of the injection of Fe in the ISM of the galaxy by SNe Ia. The intensity of the wind is very important to reproduce the abundance ratios, especially the ones with the lowest values at higher metallicities. The best model for each galaxy adopts wind efficiencies which differ considerably: while Draco and Carina requires the lowest wind efficiencies (wi $=6$ and 7 , respectively) and Sculptor the highest one (wi $=13$ ), the wind efficiency for Ursa Minor, Sextan and Sagittarius assume intermediate values (wi $=10,9$ and 9 , respectively).

\subsection{The metallicity distribution of stars}

The predicted metallicity distribution of stars of the solar vicinity compared to the ones predicted for Draco, Sagittarius, Sextan, Sculptor, Ursa Minor and Carina are shown on 

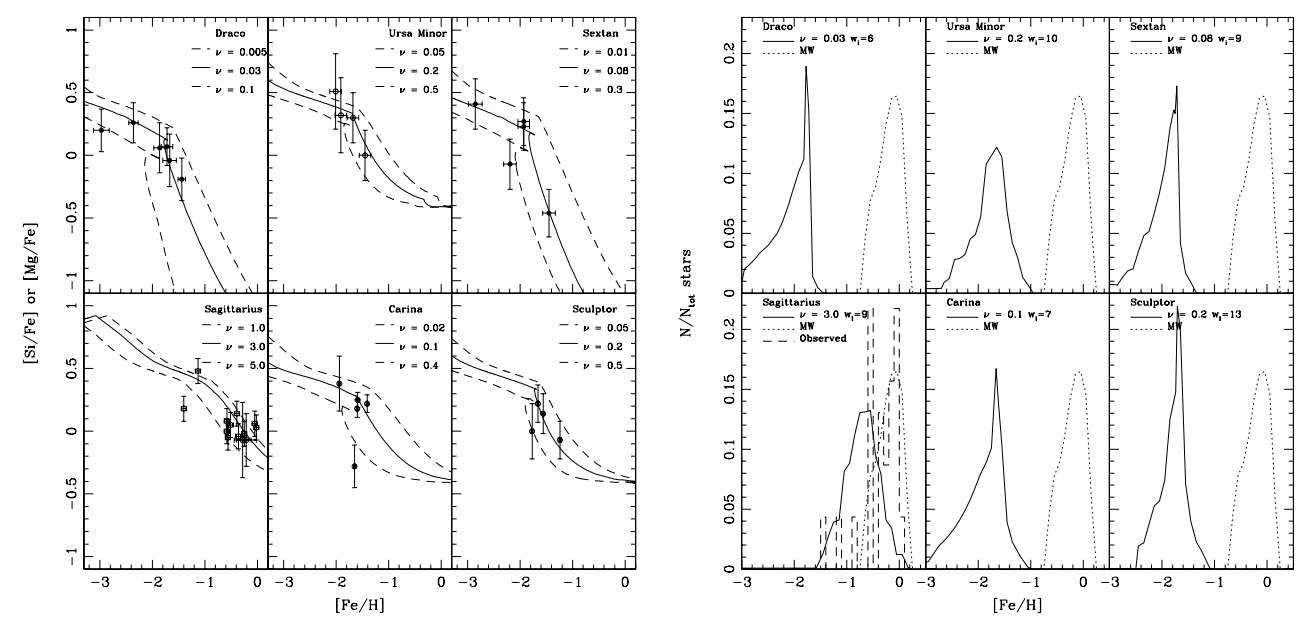

Figure 1. $[\alpha / \mathrm{Fe}]$ vs. $[\mathrm{Fe} / \mathrm{H}]$ of the models compared to observations (letf side) and the predicted metallicity distributions compared to the one of the Milky Way (right side).

the right side of Figure 1. The solid lines represent the predictions for the best model selected for each dSph galaxy and the dotted line the distribution of the model of Chiappini, Matteucci and Gratton (1997) for the solar neighbourhood, which well reproduces the observed one. In general, the peak of the metallicity distribution of the dSphs occurs at lower metallicities than the one of the solar neighborhood, but the position of the peak is specific for each galaxy, depending on the wind and SF efficiencies. The peaks at low $[\mathrm{Fe} / \mathrm{H}]$, compared to solar vicinity, are due to the lower SF efficiency and strong galactic winds. Besides that, in dSph galaxies there are several stars with metallicities well below the one of the peak (no G-dwarf problem), due to the short infall timescale assumed for dSphs.

\section{Conclusions}

The main conclusions are: five of the six dSph galaxies are characterized by very low star formation efficiencies and Sagittarius by a higher one; a high wind efficiency is required in order to reproduce the $[\alpha / \mathrm{Fe}]$ ratios and the present day mass of gas of the $\mathrm{dSph}$; the metallicity distribution of stars exhibit a peak at $[\mathrm{Fe} / \mathrm{H}] \sim-1.8$ to -1.5 dex, with the exception of Sagittarius, which shows a peak at $[\mathrm{Fe} / \mathrm{H}] \sim-0.8$ dex; the difference in the predicted peaks of the dSph galaxies and the solar vicinity is due the fact that these galaxies are characterized by much lower SF efficiencies and to the occurrence of intense galactic winds which prevent the galaxies to form stars with metallicities higher than the one of the ISM of the galaxy shortly after the time at which the wind develops; the predicted metallicity distributions of stars exhibit a peak in the same range of metallicity of the observed stars.

\section{References}

Chiappini, C., Matteucci, F., \& Gratton, R. 1997, ApJ, 477, 765

Lanfranchi, G., \& Matteucci, F. 2004, MNRAS, in press (astro-ph/0403602)

Lanfranchi, G., \& Matteucci, F. 2003, MNRAS, 345, 71 